\title{
Minimally Invasive Surgery for Intrahepatic Cholangiocarcinoma: Patient Selection and Special Considerations
}

\author{
MacKenzie L Owen' \\ Eliza W Beal (iD) ${ }^{2}$ \\ 'The Ohio State University College of \\ Medicine, Columbus, OH, USA; ${ }^{2}$ The \\ Ohio State University Comprehensive \\ Cancer Center, Department of Surgery, \\ Division of Surgical Oncology, Columbus, \\ $\mathrm{OH}$, USA
}

\begin{abstract}
Intrahepatic cholangiocarcinoma (ICC) is an aggressive primary hepatic malignancy. Unfortunately, despite advancements in diagnosis, staging and management, mortality is high. Surgery remains the only curative treatment, but many patients present with advanced, unresectable disease. For patients able to undergo surgical resection, overall survival is improved, but remains low, with high rates of disease recurrence. Minimally invasive surgery (MIS), including laparoscopic and robotic approaches, are increasingly used in surgical resection for ICC. These approaches variably demonstrate faster recovery times, less blood loss, decreased postoperative pain and fewer postoperative complications, with adequate oncologic resections. This review examines patient selection and special considerations for MIS for ICC. Patient selection is critical and includes evaluation of a patient's anatomic and oncologic resectability, as well as comorbidities.
\end{abstract}

Keywords: intrahepatic cholangiocarcinoma, minimally invasive surgery, MIS, laparoscopic surgery, robotic surgery

\section{Introduction}

Intrahepatic cholangiocarcinoma (ICC) is an aggressive primary hepatic malignancy arising from the bile duct epithelium proximal to the development of the secondary biliary branches. Behind hepatocellular carcinoma (HCC), ICC is the second most common primary liver malignancy, representing $10-15 \%$ of cases. ${ }^{1}$ Incidence of ICC has continued to increase worldwide. Though several risk factors have been identified, including chronic liver disease, biliary disorders, and parasitic infection, the cause of this increase has not been elucidated. ${ }^{2,3}$ Unfortunately, despite recent advancements in diagnosis, staging, and management of ICC, mortality remains high, with a 5-year overall survival (OS) rate of less than $10 \%{ }^{4}$ Though surgery remains the only curative treatment, over $70 \%$ of tumors are unresectable by the time of diagnosis because most patients remain asymptomatic until they develop locally advanced or late-stage disease. ${ }^{5}$ Important considerations for surgical eligibility include physiologic, anatomic and oncologic resectability - including the ability to achieve negative surgical margins, perform an adequate lymphadenectomy and leave sufficient hepatic function after resection. ${ }^{4} 5$-year OS is improved in patients able to undergo surgical resection, but remains just 20-35\%. Furthermore, disease recurs in up to $75 \%$ of patients, demonstrating the need for improved neoadjuvant and adjuvant therapies to improve long-term oncologic outcomes. ${ }^{4,6-8}$
Correspondence: Eliza W Beal

The Ohio State University Comprehensive Cancer Center, 410 W. IOth Ave, Suite 836, Columbus, OH, USA

Tel + I $614293-8000$

Fax +I 614 293-4653

Email Eliza.Beal@osumc.edu 
Minimally invasive surgery (MIS), including laparoscopic (LLR) and robotic liver resection (RLR), is increasingly used for resection of liver lesions, including benign conditions, HCC, colorectal liver metastasis, and ICC. ${ }^{9}$ However, MIS is less commonly used than open liver resection (OLR) in the treatment of ICC. ${ }^{10}$ MIS approaches demonstrate decreased estimated blood loss (EBL), faster recovery times, decreased postoperative pain, and fewer postoperative complications than open approaches to liver resection, while demonstrating no difference in short-term morbidity or mortality or in longterm OS. ${ }^{1,9,11-17}$ Few studies assess RLR for ICC. ${ }^{18,19}$ In this review, we examine patient selection and special considerations for MIS in the treatment of ICC.

\section{Patient Selection Preoperative Evaluation Diagnosis}

Diagnosing ICC remains a challenge because patients are often asymptomatic. The most common presenting symptom of ICC is jaundice, although this occurs in only 10 $15 \%$ of cases. ${ }^{20}$ Other symptoms are nonspecific and include abdominal pain, weight loss, weakness, and malaise. In up to $25 \%$ of cases, ICC is diagnosed as an incidental finding on imaging. ${ }^{20}$ Workup for suspected ICC can be directed by the National Comprehensive Cancer Network (NCCN) guidelines, which recommend history and physical exam, imaging, laboratory tests, esophagogastroduodenoscopy (EGD), colonoscopy, and surgical consultation with a multidisciplinary team. ${ }^{21}$ Imaging should consist of multiphasic abdominopelvic computed tomography (CT) or magnetic resonance imaging (MRI) with intravenous (IV) contrast and chest CT with or without contrast. Laboratory tests include liver function tests (LFTs), viral hepatitis serologies, and assessment of tumor markers, such as carcinoembryonic antigen (CEA), cancer antigen 19-9 (Ca 19-9), and alpha-fetoprotein (AFP). According to the NCCN, a preoperative biopsy is not required for patients with suspected malignancy on imaging before resection with curative intent. In practice, ICC can be difficult to distinguish from other adenocarcinomas, and workup for other primary malignancies can be pursued in cases where the diagnosis is uncertain. For ICC patients being evaluated for surgical resection, the NCCN recommends considering staging laparoscopy to assess for multifocal hepatic disease and distant lymph node tumor metastases, as these are contraindications to resection.

\section{Imaging}

The most common presenting symptom of ICC is jaundice, which is often initially evaluated by US. US is useful in excluding benign etiologies of obstructive jaundice and can differentiate ICC from HCC when contrast agents are added. Defining features of ICC on contrast-enhanced (CE) US include irregular peripheral rim-like enhancement in arterial phase as well as faster arterial hyperenhancement and contrast washout in ICC than HCC. ${ }^{22}$

Multiphase CE-CT is the standard imaging modality for diagnosis, staging, and prognostic evaluation of ICC. ${ }^{23}$ Like CE-US, CE-CT can distinguish ICC from HCC through differences in appearance on arterial, venous, and delayed contrast phases. ICC often displays peripheral rim-like enhancement with central hypovascularity during arterial and venous phases with progressive incomplete centripetal enhancement on CE-CT. ${ }^{23,24}$ This reflects the morphologic distribution of malignant cells at the periphery of the tumor, and may have prognostic value because central hypovascularity is associated with increased lymphatic and biliary invasion. ${ }^{23,25}$ Further, ICC demonstrates enhancement of fibrous stroma in the delayed phase, a distinctive feature of cholangiocarcinoma. ${ }^{25}$ In contrast, HCC tends to display early arterial enhancement with washout in the portal or delayed phases. ${ }^{24} \mathrm{CT}$ is also useful for anatomic evaluation of the tumor and nearby structures for resection planning. ${ }^{23}$

MRI is increasingly used for diagnosis and staging of ICC due to its superior ability to detect biliary tumor spread and lymph node involvement in comparison to CT. ${ }^{25}$ MRI for workup of ICC should include T1- and T2weighted images, diffusion-weighted imaging (DWI), triphasic $\mathrm{CE}$ sequences and $\mathrm{T} 2$-weighted magnetic resonance cholangiopancreatography (MRCP) to noninvasively assess the biliary tree. ${ }^{25}$ Mass-forming ICC displays lowintensity T1 signal, high-intensity T2 signal, and a "target appearance" of peripheral rim enhancement and progressive centripetal enhancement on DWI. ${ }^{24}$ DWI can also help identify malignant biliary strictures, an essential step in diagnosing periductal-infiltrating ICC. ${ }^{25}$ In general, CEMRI features of ICC are similar to those on CE-CT and are again helpful in differentiating ICC from HCC, which demonstrates arterial hyperenhancement, venous capsular enhancement, and intralesional fat. ${ }^{24}$

ICC occurs in three macroscopic growth patterns: mass-forming, periductal-infiltrating, and intraductalgrowth. ${ }^{25}$ The mass-forming type is most common and 
presents as an unencapsulated, tan liver mass. In contrast, the periductal-infiltrating type can cause biliary strictures or fibrosis, and the intraductal-growth type grows intraluminally. $^{23}$ These subtypes vary in appearance on imaging and impact the sensitivity and specificity of different imaging tests, including ultrasound (US), CT, MRI, and positron emission tomography (PET).

PET-CT is a valuable tool in identifying ICC, with a sensitivity and specificity of over $90 \%$, compared to $60 \%$ for diagnosing extrahepatic cholangiocarcinoma. It has also demonstrated improved detection of nodal metastases over CT and MRI, resulting in changes in clinical management in up to $30 \%$ of patients, and therefore may be considered as an additional modality in preoperative assessment of ICC. ${ }^{25}$

\section{Preoperative Evaluation}

Preoperative evaluation of surgical candidates may initially include consultation with anesthesia and medicine services, such as cardiology and hepatology, for assessment of hepatic function, cardiovascular disease, and pulmonary status. ${ }^{26}$ Hepatic function is defined in terms of the future liver remnant (FLR), which constitutes the percentage of intact hepatic arterial, portal venous, hepatic venous and biliary flow after resection. The typical recommendation is that adequate FLR is $>20 \%$ in individuals with normal liver function and up to $50 \%$ in those with severe preexisting liver disease. ${ }^{26}$ However, if expected FLR is insufficient, induction of liver hypertrophy to increase FLR with portal vein embolization, liver venous deprivation, or associated liver partition and portal vein ligation (ALPPS) can be considered. ${ }^{4}$ Cardiovascular status should also be assessed preoperatively for patients undergoing noncardiac surgeries. Cardiovascular functional status is defined by metabolic equivalents (METs) based on activities of daily living. Asymptomatic patients with low surgical risk or $\geq 4$ METs may proceed with surgery without further cardiac testing. For those with poor functional capacity of $<4$ METs, the American College of Cardiology recommends evaluation with stress echocardiography. ${ }^{27}$ Cardiac stressors specific to LLR should also be considered, including hemodynamic changes due to abdominal insufflation, reverse Trendelenburg positioning, and intraoperative vascular occlusion, especially in patients with underlying cardiovascular disease. ${ }^{26}$ Pulmonary complications are reduced in LLR relative to OLR; therefore, standard evaluation relative to the patient's underlying pulmonary status is sufficient. $^{28}$

\section{Multidisciplinary Care Discussion}

It is essential to consider a multidisciplinary (MD) approach to care for the management of patients with ICC, which may include surgical oncology, medical oncology, radiation oncology, transplant surgery, hepatology, radiology, and pathology. While studies examining ICC outcomes with MD care are limited, research has shown that MD liver tumor boards effectively diagnose and stage HCC. Furthermore, patients receiving MD care have more individualized treatment strategies. ${ }^{29}$ For example, Lau et al conducted a study at Veterans Affairs medical centers in Texas that showed an increase in referrals, surgical procedures, and use of perioperative chemotherapy after the establishment of a MD hepatopancreaticobiliary surgical program. ${ }^{30}$ This study also demonstrated a decrease in postoperative complications, 90-day mortality, and length of hospital stay. ${ }^{30}$ Additionally, Salami et al found that establishing a MD virtual tumor board led to an increase in the number of HCC patients receiving a MD evaluation, which occurred at faster rates and with a decreased travel burden for patients. $^{31}$ Given these improvements to patients' care process and treatment strategies, MD evaluation should be employed when possible in the management of ICC.

\section{Indications for Surgery}

Surgical resection of ICC is indicated when microscopically (R0) negative margins can be safely achieved. ${ }^{21}$ Evaluation for surgical resectability takes into account physiologic, anatomic, and oncologic factors. Physiologic resectability considers a patient's comorbidities and functional status to determine their ability to tolerate a major operative procedure and is assessed with a thorough preoperative evaluation. $^{26}$ Anatomic resectability requires removal of the diseased liver segments while maintaining sufficient FLR, which can in part be determined by preoperative liver volumetry. ${ }^{26}$ Oncologic resectability requires consideration of multiple factors, including the degree of liver involvement, presence of lymph node or vascular involvement, levels of tumor markers, and response to prior therapies. ICC staging is an essential first step to determine the extent of tumor spread. ${ }^{32,33}$ Staging can be performed with recommendations from the 8th edition of the American Joint Committee on Cancer/International Union against Cancer (AJCC/UICC) 
consensus statement. This system has been validated in its prognostic value for OS. According to NCCN and AJCC/ UICC guidelines, surgical resection is generally contraindicated in the presence of multifocal liver disease or extrahepatic involvement because these factors indicate metastasis. $^{21,32,33}$ Similarly, lymph node metastasis to the porta hepatis and involvement beyond the regional lymph nodes or evidence of microvascular invasion generally make a tumor inoperable. However, resection may be considered in select cases by experienced surgeons. ${ }^{21,34}$ In patients with high-risk for occult metastasis, including those with elevated Ca 19-9 levels, suspected microvascular invasion, or signs of peritoneal disease, a staging laparoscopy should be considered. Up to $36 \%$ of these otherwise surgical candidates have unresectable disease on staging laparoscopy. ${ }^{32}$ Of patients ultimately eligible for surgical resection, recurrence is common, especially among those with a large primary tumor size $>5 \mathrm{~cm}$, multiple lesions, cirrhosis, and lymph node or vascular involvement. ${ }^{6,32}$ In patients with intrahepatic recurrence, a subset up to $25 \%$ may be considered for repeat liver resection, which has demonstrated improved OS compared to other approaches, such as ablation, intra-arterial therapy, or supportive care. Chemotherapy has also shown improved OS compared to supportive care after recurrence. ${ }^{34,35}$

\section{Minimally Invasive Surgery MIS Liver Resection}

MIS, encompassing both LLR and RLR, has recently gained traction as a surgical approach to treating various liver diseases, including benign and malignant conditions. LLR is indicated for the management of benign liver tumors when symptomatic, at risk for rupture, or in the case of diagnostic uncertainty. Use of LLR has also expanded to include management of malignant liver tumors, especially HCC, ICC, and colorectal liver metastases. The laparoscopic approach requires similar considerations as OLR, such as the ability to tolerate surgery, especially from a cardiopulmonary perspective, and the determination of FLR. ${ }^{9}$ In the early days of LLR, the Louisville Statement, published in 2008, guided providers in selecting appropriate patients for MIS resection including those with tumor size $<5 \mathrm{~cm}$ and located in segments $2-6 .{ }^{36}$ More recently surgeons at experienced centers have demonstrated the safety and feasibility of laparoscopic right hepatectomy. ${ }^{37-39}$
Studies have consistently shown that LLR provides the following advantages compared to OLR: decreased blood loss, lower rate of transfusions, shorter hospital stay, decreased postoperative pain and fewer postoperative complications. $^{4,9,40}$ Further, in the setting of malignancy, specifically colorectal metastases and ICC, LLR has demonstrated decreased intra-operative blood loss, improved short-term outcomes, and similar long-term oncologic outcomes compared to OLR. ${ }^{9,10,41}$

Similar to LLR, RLR is indicated for surgical management of various benign and malignant hepatic tumors. ${ }^{42}$ Additionally, benefits of RLR include surgeon comfort, decreased blood loss, shorter hospital stay, and improved short-term outcomes compared to OLR or hand-assisted techniques. Unlike LLR, RLR is not technically limited by tumors in the posterosuperior position. Limitations of RLR include technical complexity, increased difficulty controlling bleeding, longer operative times, and higher surgical costs than LLR. ${ }^{42,43}$

\section{MIS Liver Resection for ICC}

Surgical resection of ICC typically involves liver resection and lymphadenectomy (LAD). In contrast, perihilar cholangiocarcinoma (PCC) often requires lobectomy with bile duct resection, LAD, and Roux-en-Y hepaticojejunostomy, and distal cholangiocarcinoma (DCC) requires pancreaticoduodenectomy (Whipple procedure). ${ }^{44}$ With MIS approaches gaining traction in liver resections, there has been increased interest in using LLR for surgical management of ICC, though data assessing this approach is still relatively limited. Even more limited data exists for RLR.

Early studies of LLR for ICC demonstrated decreased EBL, decreased Pringle maneuver time, and no differences in perioperative transfusions, OT, perioperative morbidity, perioperative mortality, RFS or OS (Table 1). ${ }^{1,11,12}$ Most studies also demonstrate no difference in rate of lymphadenectomy or number of lymph nodes harvested between LLR or OLR, however one early study did demonstrate an increase in rate of lymphadenectomy in OLR. ${ }^{1,11,12}$ Importantly, in a study of patients with large $(\geq 5 \mathrm{~cm})$ or multiple ( $\geq 2$ ) ICC, Zhu et al demonstrated that after propensity score matching (PSM), LLR resulted in longer operating room time (OT), and Pringle maneuver time, but that there was no difference in EBL, LOS, grade III/ IV complication rate, DFS or OS. ${ }^{13}$ In a further study, Wei et al compared patients undergoing LLR for large $(\geq 5 \mathrm{~cm})$ or multiple $(\geq 2)$ ICCs $(\mathrm{N}=12)$, patients who underwent LLR for small solitary ICCs $(\mathrm{N}=18)$, and patients who 


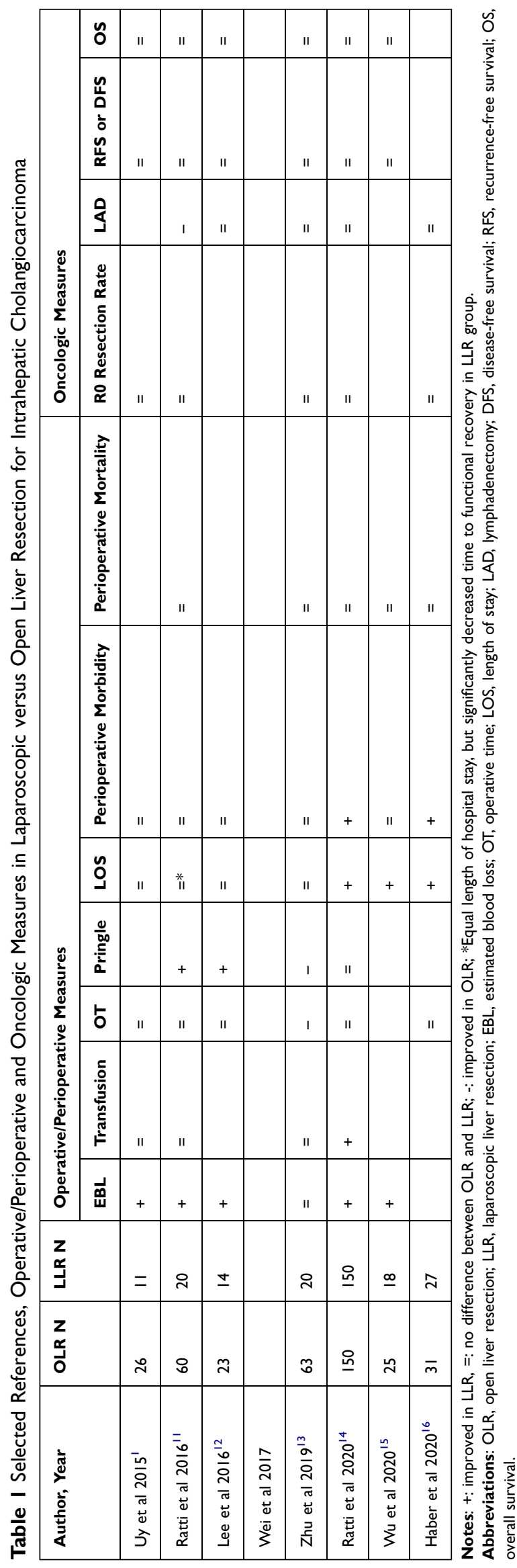

underwent LLR for large or multiple ICCs $(\mathrm{N}=20)$ noting that perioperative and long-term outcomes were similar between all three groups of patients. The authors concluded that LLR for large or multiple ICCs is feasible and safe and maintains oncologic effectiveness. ${ }^{45}$

Among the more recently published single center studies, results are more variable, but have started to show improvements in EBL, LOS, perioperative morbidity including blood transfusions in LLR, with no differences in oncologic outcomes such as R0 resection, rate of lymphadenectomy and DFS/RFS or OS (Table 1). ${ }^{14-16}$ One demonstrated lower EBL, fewer intraoperative and postoperative transfusions, shorter LOS, and reduced perioperative morbidity in LLR compared to OLR with no difference in OT, Pringle maneuver time, perioperative mortality, R0 resection rate, rate of lymphadenectomy, DFS or OS. ${ }^{14}$ A second demonstrated an improvement in EBL and LOS with LLR, with no difference in perioperative morbidity or mortality or long-term RFS/DFS or OS. ${ }^{15} \mathrm{~A}$ third demonstrated an improvement in LOS and perioperative morbidity with no change in OT, R0 resection rate or rate of lymphadenectomy, but did not report long-term outcomes such as RFS/DFS or OS. ${ }^{16}$

Evidence regarding RLR for intrahepatic cholangiocarcinoma is very limited.

\section{Conclusion}

Minimally invasive surgery is safe and feasible for resection of ICC. It has been demonstrated that LLR demonstrates faster recovery time, decreased blood loss, decreased postoperative pain and fewer postoperative complications, with adequate oncologic resections including lymphadenectomy and rates of $\mathrm{R} 0$ resection in patients with ICC in small studies. In the future, the increased use of LLR for resection of ICC will allow for confirmation of these important benefits, and further elucidation of any additional detriment. Prospective and randomized studies could be considered. Importantly, patient selection is critical including assessment of patient comorbidities, anatomic and oncologic resectability. More investigation into RLR of ICC is needed.

\section{Abbreviations}

ICC, intrahepatic cholangiocarcinoma; HCC, hepatocellular carcinoma; OS, overall survival; MIS, minimally invasive surgery; LLR, laparoscopic liver resection; RLR, robotic liver resection; OLR, open liver resection; EBL, estimated blood loss; NCCN, National Comprehensive 
Cancer Network; EGD, esophagogastroduodenoscopy; CT, computed tomography; MRI, magnetic resonance imaging; IV, intravenous; LFTs, liver function tests; CEA, carcinoembryonic antigen; Ca 19-9, cancer antigen 19-9; AFP, alpha-fetoprotein; US, ultrasound; PET, positron emission tomography; CE, contrast-enhanced; DWI, diffusion weighted imaging; MRCP, magnetic resonance cholangiopancreatography; FLR, future liver remnant; MET, metabolic equivalents; MD, multidisciplinary; AJCC/UICC, American Joint Committee on Cancer/International Union against Cancer; LAD, lymphadenectomy; PCC, perihilar cholangiocarcinoma; DCC, distal cholangiocarcinoma; LOS, length of stay; OT, operating room time.

\section{Acknowledgments}

The authors have no acknowledgements.

\section{Disclosure}

The authors report no conflicts of interest in this work.

\section{References}

1. Uy BJ, Han HS, Yoon YS, Cho JY. Laparoscopic liver resection for intrahepatic cholangiocarcinoma. J Laparoendosc Adv Surg Tech. 2015;25(4):272-277. doi:10.1089/lap.2014.0233

2. Beal EW, Tumin D, Moris D, et al. Cohort contributions to trends in the incidence and mortality of intrahepatic cholangiocarcinoma. Hepatobiliary Surg Nutr. 2018;7(4):270-276. doi:10.21037/hbsn.20 18.03.16

3. Bergquist A, Von Seth E. Epidemiology of cholangiocarcinoma. Best Pract Res Clin Gastroenterol. 2015;29(2):221-232. doi:10.1016/j. bpg.2015.02.003

4. Beal EW, Cloyd JM, Pawlik TM. Surgical treatment of intrahepatic cholangiocarcinoma: current and emerging principles. J Clin Med. 2020;10(1):104. doi:10.3390/jcm10010104

5. Zhai ST, Liang X, Mao QJ, et al. A retrospective pilot study to examine the feasibility of real-time navigation for laparoscopic liver resections in intrahepatic cholangiocarcinoma using fusion indocyanine green fluorescence imaging. J Surg Oncol. 2020;122(2):2 26-233. doi: $10.1002 /$ jso. 25940

6. Zhang X-F, Beal EW, Bagante F, et al. Early versus late recurrence of intrahepatic cholangiocarcinoma after resection with curative intent. Br J Surg. 2018;105(7):848-856.

7. Rizzo A, Brandi G. Pitfalls, challenges, and updates in adjuvant systemic treatment for resected biliary tract cancer. Expert Rev Gastroenterol Hepatol. 2021;15(5):547-554. doi:10.1080/17474124. 2021.1890031

8. Rizzo A, Brandi G. Neoadjuvant therapy for cholangiocarcinoma: a comprehensive literature review. Cancer Treat Res Commun. 2021;27:100354. doi:10.1016/j.ctarc.2021.100354

9. Cho JY, Han HS, Wakabayashi G, et al. Practical guidelines for performing laparoscopic liver resection based on the second international laparoscopic liver consensus conference. Surg Oncol. 2018;27 (1):A5-9. doi:10.1016/j.suronc.2017.12.003

10. Guerrini GP, Esposito G, Tarantino G, et al. Laparoscopic versus open liver resection for intrahepatic cholangiocarcinoma: the first meta-analysis. Langenbecks Arch Surg. 2020;405(3):265-275. doi:10.1007/s00423-020-01877-0
11. Ratti F, Cipriani F, Ariotti R, et al. Safety and feasibility of laparoscopic liver resection with associated lymphadenectomy for intrahepatic cholangiocarcinoma: a propensity score-based case-matched analysis from a single institution. Surg Endosc. 2016;30 (5):1999-2010. doi:10.1007/s00464-015-4430-4

12. Lee W, Park JH, Kim JY, et al. Comparison of perioperative and oncologic outcomes between open and laparoscopic liver resection for intrahepatic cholangiocarcinoma. Surg Endosc. 2016;30 (11):4835-4840. doi:10.1007/s00464-016-4817-x

13. Zhu Y, Song J, Xu X, Tan Y, Yang J. Safety and feasibility of laparoscopic liver resection for patients with large or multiple intrahepatic cholangiocarcinomas: a propensity score based case-matched analysis from a single institute. Med (United States). 2019;98(49): e18307.

14. Ratti F, Rawashdeh A, Cipriani F, et al. Intrahepatic cholangiocarcinoma as the new field of implementation of laparoscopic liver resection programs. A comparative propensity score-based analysis of open and laparoscopic liver resections. Surg Endosc. 2020. doi:10.1007/s00464-020-07588-3

15. Wu J, Han J, Zhang Y, et al. Safety and feasibility of laparoscopic versus open liver resection with associated lymphadenectomy for intrahepatic cholangiocarcinoma. Biosci Trends. 2020;14:376-383. doi:10.5582/bst.2020.03293

16. Haber PK, Wabitsch S, Kästner A, et al. Laparoscopic liver resection for intrahepatic cholangiocarcinoma: a single-center experience. J Laparoendosc Adv Surg Tech. 2020;00:1-6.

17. Ratti F, Rawashdeh A, Cipriani F, et al. Intrahepatic cholangiocarcinoma as the new field of implementation of laparoscopic liver resection programs. A comparative propensity score-based analysis of open and laparoscopic liver resections. Surg Endosc. 2021;35 (4):1851-1862. doi:10.1007/s00464-020-07588-3

18. Di Benedetto F, Magistri P. First case of full robotic ALPPS for intrahepatic cholangiocarcinoma. Ann Surg Oncol. 2020;10434. doi:10.1245/s10434-020-08794-y

19. Levi Sandri GB, Spoletini G, Mascianà G, et al. The role of minimally invasive surgery in the treatment of cholangiocarcinoma. Eur J Surg Oncol. 2017;43(9):1617-1621. doi:10.1016/j.ejso.2017.02.012

20. Forner A, Vidili G, Rengo M, Bujanda L, Ponz-Sarvisé M, Lamarca A. Clinical presentation, diagnosis and staging of cholangiocarcinoma. Liver Int. 2019;39(S1):98-107. doi:10.1111/liv.14086

21. NCCN. NCCN clinical practice guidelines in oncology (NCCN guidelines): hepatobiliary cancers, version 4.2021 - August 26, 2021; 2021. Available from: https://www.nccn.org/professionals/phy sician_gls/pdf/hepatobiliary.pdf. Accessed December 16, 2021.

22. Liu GJ, Wang W, De LM, et al. Contrast-enhanced ultrasound for the characterization of hepatocellular carcinoma and intrahepatic cholangiocarcinoma. Liver Cancer. 2015;4(4):241-252. doi:10.1159/ 000367738

23. Joo I, Lee JM, Yoon JH. Imaging diagnosis of intrahepatic and perihilar cholangiocarcinoma: recent advances and challenges. Radiology. 2018;288(1):7-23. doi:10.1148/radiol.2018171187

24. Seo N, Kim DY, Choi JY. Cross-sectional imaging of intrahepatic cholangiocarcinoma: development, growth, spread, and prognosis. Am J Roentgenol. 2017;209(2):W64-75. doi:10.2214/AJR.16.16923

25. Fábrega-Foster K, Ghasabeh MA, Pawlik TM, Kamel IR. Multimodality imaging of intrahepatic cholangiocarcinoma. HepatoBiliary Surg Nutr. 2017;6(2):67-78. doi:10.21037/hbsn.2016.12.10

26. Egger ME, Gottumukkala V, Wilks JA, et al. Anesthetic and operative considerations for laparoscopic liver resection. Surg (United States). 2017;161(5):1191-1202. doi:10.1016/j.surg.2016.07.011

27. Fleisher LA, Fleischmann KE, Auerbach AD, et al. 2014 ACC/AHA guideline on perioperative cardiovascular evaluation and management of patients undergoing noncardiac surgery: a report of the American college of cardiology/American heart association task force on practice guidelines. J Am Coll Cardiol. 2014;64(22):e77137. doi:10.1016/j.jacc.2014.07.944 
28. Fuks D, Cauchy F, Fteriche S, et al. Laparoscopy decreases pulmonar $\mathrm{y}$ complications in patients undergoing major liver resection a propensity score analysis. Ann Surg. 2016;263(2):353-361. doi:10.1097/SLA.0000000000001140

29. Siddique O, Yoo ER, Perumpail RB, et al. The importance of a multidisciplinary approach to hepatocellular carcinoma. $J$ Multidiscip Healthc. 2017;10:95-100. doi:10.2147/JMDH.S12 8629

30. Lau K, Salami A, Barden G, et al. The effect of a regional hepatopancreaticobiliary surgical program on clinical volume, quality of cancer care, and outcomes in the veterans affairs system. JAMA Surg. 2014;149(11):1153-1161. doi:10.1001/jamasurg.2014.1711

31. Salami AC, Barden GM, Castillo DL, et al. Establishment of a regional virtual tumor board program to improve the process of care for patients with hepatocellular carcinoma. J Oncol Pract. 2015;11(1):E66-74. doi:10.1200/JOP.2014.000679

32. Weber SM, Ribero D, O’Reilly EM, Kokudo N, Miyazaki M, Pawlik TM. Intrahepatic cholangiocarcinoma: expert consensus statement. Hpb. 2015;17(8):669-680. doi:10.1111/hpb.12441

33. Lee AJ, Chun YS. Intrahepatic cholangiocarcinoma: the AJCC/UICC 8th edition updates. Chin Clin Oncol. 2018;7(5):9-11. doi:10.21037/ cco.2018.07.03

34. Machairas N, Lang H, Jayant K, Raptis DA, Sotiropoulos GC. Intrahepatic cholangiocarcinoma: limitations for resectability, curren surgical concepts and future perspectives. Eur J Surg Oncol. 2020;46 (5):740-746. doi:10.1016/j.ejso.2020.01.028

35. Spolverato G, Kim Y, Alexandrescu S, et al. Management and outcomes of patients with recurrent intrahepatic cholangiocarcinoma following previous curative-intent surgical resection. Ann Surg Oncol. 2016;23(1):235-243. doi:10.1245/s10434-015-4642-9

36. Buell JF, Cherqui D, Geller DA, et al. The international position on laparoscopic liver surgery. Ann Surg. 2009;250(5):825-830. doi:10. 1097/SLA.0b013e3181b3b2d8
37. Hilal MA, Di Fabio F, Teng MJ, Lykoudis P, Primrose JN, Pearce NW. Single-centre comparative study of laparoscopic versus open right hepatectomy. J Gastrointest Surg. 2011;15(5):818-823. doi:10.1007/s11605-011-1468-z

38. Dagher I, Caillard C, Proske JM, Carloni A, Lainas P, Franco D. Laparoscopic right hepatectomy: original technique and results. $J \mathrm{Am}$ Coll Surg. 2008;206(4):756-760. doi:10.1016/j.jamcollsurg.2007.09.012

39. Pearce NW, Di Fabio F, Teng MJ, Syed S, Primrose JN, Abu Hilal M Laparoscopic right hepatectomy: a challenging, but feasible, safe and efficient procedure. Am J Surg. 2011;202(5):e52-e58. doi:10.1016/j. amjsurg.2010.08.032

40. Ciria R, Cherqui D, Geller DA, Briceno J, Wakabayashi G. Comparative short-term benefits of laparoscopic liver resection: 9000 cases and climbing. Ann Surg. 2016;263(4):761-777. doi:10.1097/SLA.0000000000001413

41. Hilal MA, Aldrighetti L, Dagher I, et al. The Southampton consensus guidelines for laparoscopic liver surgery: from indication to implementation. Ann Surg. 2018;268(1):11-18. doi:10.1097/SLA. 0000000000002524

42. Lafaro KJ, Stewart C, Fong A, Fong Y. Robotic liver resection. Surg Clin North Am. 2020;100(2):265-281. doi:10.1016/j.suc.2019.11.003

43. Mejia A, Cheng SS, Vivian E, Shah J, Oduor H, Archarya P. Minimally invasive liver resection in the era of robotics: analysis of 214 cases. Surg Endosc. 2020;34(1):339-348. doi:10.1007/s00464019-06773-3

44. Rizvi S, Khan SA, Hallemeier CL, Kelley RK, Gores GJ. Cholangiocarcinoma-evolving concepts and therapeutic strategies. Nat Rev Clin Oncol. 2018;15(2):95-111. doi:10.1038/nrclinonc.20 17.157

45. Wei F, Lu C, Cai L, Yu H, Liang X, Cai X. Can laparoscopic liver resection provide a favorable option for patients with large or multiple intrahepatic cholangiocarcinomas? Surg Endosc. 2017;31 (9):3646-3655. doi:10.1007/s00464-016-5399-3
Hepatic Medicine: Evidence and Research

\section{Publish your work in this journal}

Hepatic Medicine: Evidence and Research is an international, peerreviewed, open access journal covering all aspects of adult and pediatric hepatology in the clinic and laboratory including the following topics: Pathology, pathophysiology of hepatic disease; Investigation and treatment of hepatic disease; Pharmacology of drugs used for the treatment of hepatic disease. Issues of patient safety and quality of care will also be considered. The manuscript management system is completely online and includes a very quick and fair peer-review system, which is all easy to use. Visit http://www.dovepress.com/ testimonials.php to read real quotes from published authors. 